\title{
Courtiers en développement et contrôleurs d'accès: deux figures incontournables des politiques de lutte contre la pauvreté rurale en Afrique du Sud
}

\author{
Magalie Bourblanc ${ }^{1,2,3, *}$ et Raphaëlle Ducrot ${ }^{1,2}$ \\ ${ }^{1}$ Cirad, UMR G-EAU, 34398 Montpellier, France \\ 2 G-EAU, Université de Montpellier, Montpellier, France \\ ${ }^{3}$ Centre for the Study of Governance Innovation (GovInn), University of Pretoria, Pretoria, Afrique du Sud
}

\begin{abstract}
Résumé - Cet article analyse la mise en œuvre de programmes publics de lutte contre la pauvreté rurale en Afrique du Sud et fait le parallèle avec les politiques d'aide internationale au développement, en soulignant en particulier un de leurs travers, à savoir leur relative inefficacité. En remobilisant un concept-clé des travaux en anthropologie, celui du courtier, couplé à la redécouverte de l'idée de "point de passage obligé » (gatekeeping) issue de la science politique, cet article montre à quel point les courtiers ont l'ambition de s'imposer comme des intermédiaires hégémoniques au sein d'un univers du courtage très disputé. À cette occasion, notre article s'interroge sur les relations entre les figures nouvelles et traditionnelles du courtage, c'est-à-dire entre les conseils d'administration des comités d'irrigation et les élites tribales.
\end{abstract}

Mots clés : courtiers en développement / contrôleurs d'accès / politiques publiques / développement rural / Afrique du Sud

\begin{abstract}
Development brokers and gatekeepers: two critical figures of public policies tackling rural poverty in South Africa. This paper studies the implementation of national public policies tackling rural poverty in South Africa and draws a parallel with international development aid public policies, stressing in particular one of their common predicaments, i.e. their relative ineffectiveness. Building on the key notion of "brokerage" in anthropology, and coupling it with the notion of "gatekeeping" derived from the political science literature, this paper demonstrates how much brokers strive to become hegemonic intermediaries within a very competitive brokerage environment. This paper questions the relations between new and pre-existing figures of brokerage, i.e. between an irrigation committee's governing board and village's traditional elites.
\end{abstract}

Keywords: development brokers / gatekeepers / public policies / rural development / South Africa

\section{Introduction}

Dans son ouvrage canonique The anti-politics machine, Ferguson (1990) met en évidence les écueils des programmes internationaux d'aide publique au développement, notamment leur manque de considération pour le contexte local d'intervention (historique, social, politique, économique, etc.). L'étude de programmes publics nationaux censés favoriser l'accès à l'eau et au foncier pour les populations rurales pauvres d'Afrique du Sud révèle les mêmes travers. En effet, dans les pays du Sud, les programmes publics d'origine nationale dirigés vers les zones rurales, très souvent sous-

\footnotetext{
$\overline{\text { *Auteur de correspondance }}$ : magalie.bourblanc@cirad.fr
}

développées, présentent souvent des caractéristiques similaires aux politiques internationales d'aide au développement initiées par des bailleurs de fonds ou par des pays développés dans le cadre de leurs politiques de coopération. Plus d'une fois, ils sont sans effet, parfois encore ils aboutissent au résultat inverse de celui escompté. Rares sont ceux qui atteignent leurs cibles.

Cet article se propose de rapprocher ces deux littératures que sont la littérature sur l'aide internationale au développement et celle relative à l'analyse des politiques publiques (nationales). D'abord perçue comme une politique internationale, l'aide internationale au développement des pays du Sud a été trop peu souvent analysée comme une politique publique à part entière. Pourtant, certains auteurs soulignent à raison que, de par ses nouvelles modalités d'intervention - de moins en moins directes et de plus en plus imbriquées avec les 
acteurs nationaux de politiques publiques depuis la déclaration de Paris (2005) -, cette aide internationale au développement devrait se concevoir comme telle, à savoir comme une politique publique nationale (Smith, 2013). En outre, l'extraversion des États dits du Sud et de leurs politiques publiques - c'est-à-dire leur forte dépendance vis-à-vis de l'extérieur et des pays dits développés en particulier - n'est plus à démontrer (Bayart et al., 1992), même s'il convient de distinguer au sein des pays du Sud ceux qui sont les plus marqués par l'influence des bailleurs de fonds internationaux.

La littérature anthropologique francophone et les travaux de Bierschenk et al. (2000) en particulier font également écho à cette volonté de rapprochement entre traditions académiques. La «socioanthropologie des espaces publics africains» (Olivier de Sardan, 2007) de ces anthropologues francophones offre une analyse qui entre en résonance avec l'analyse de l'action publique dans différentes régions d'Afrique (Darbon, 2004) et l'analyse du politique par le bas (Bayart et al., 1992). Ces anthropologues ont développé en particulier le concept de «courtier en développement», qui fait écho au concept de broker, issu de la littérature anthropologique africaine d'origine anglo-saxonne dont ils s'inspirent. À l'origine, les travaux anglo-saxons, alors dominés par le structuralofonctionnalisme, étaient plus prompts à parler d'«intermédiaires » que de courtiers, dans la mesure où ils envisageaient un rôle essentiellement passif pour ces acteurs durant la période coloniale (Gluckman et al., 1949). Ce sont les chefs traditionnels qui représentaient ces intermédiaires dans un contexte de faiblesse de l'État et de manque de légitimité, les élites colonisatrices devant se reposer sur des relais de leur action dans les territoires reculés qu'elles ne pouvaient atteindre. Plus tard, dans les années 1960-1970, cette tradition anglo-saxonne travaillant sur les «courtiers politiques» (political brokers) a plus insisté sur les marges de manœuvre que possédaient ces acteurs, qui étaient loin d'être de simples relais de la volonté des puissances colonisatrices ou, dans le contexte actuel, des agences internationales de développement. Le courtage a alors été associé au clientélisme et à une forme de corruption, à l'idée que le courtage et les relations protecteur-client (patron-client) étaient inextricablement liés (Bailey, 1960; Mair, 1968; Boissevain, 1969, 1974). En cela, cette seconde génération de travaux sur les brokers peut être rapprochée du concept de gatekeeper (gardien), développée par les travaux en histoire (Cooper, 2002) et en science politique pour l'Afrique du Sud notamment (Beresford, 2015; Evans, 2012). Pour la littérature sur le «courtage politique», les élites traditionnelles étaient l'archétype de ces "gardiens contrôlant l'accès au terrain », sorte de maîtres du jeu institutionnel local, situés dans des positions stratégiques, d'autant plus quand ces chefs «traditionnels» devaient leur nouvelle position aux puissances coloniales qui les avaient installés (Mamdani, 1996). S'inscrivant pleinement dans cette filiation anglo-saxonne, les travaux de Bierschenk et al. (2000) ont le mérite d'avoir également mis en exergue le rôle de nouveaux acteurs, dans un contexte post-colonial, notamment les comités villageois présentés comme des courtiers à part entière. Ils ont en particulier insisté sur la forte capacité d'initiative de ces acteurs, dont l'une des principales activités est de faire la chasse aux projets de développement. Ces travaux ont été prolongés depuis par Mosse et Lewis (2006), qui mettent en avant, quant à eux, le travail de traduction effectué par ces courtiers dans un contexte marqué par le pluralisme juridique, un pouvoir polycéphale et des empilements institutionnels peu clairs ou contestés (Mosse et Lewis, 2006).

Cette dimension plus positive du rôle de courtier ne saurait éclipser ce qui constitue en fait l'activité première d'un courtier, à savoir le fait qu'il organise à son profit un système de barrières et de passages obligés dont lui seul contrôle l'accès. Ainsi, en combinant la notion de « courtier » avec celle de «contrôleur d'accès", on entend montrer que ces « entrepreneurs » occupent des positions-clés du dispositif et qu'on ne saurait les décrire comme des intermédiaires parmi d'autres, intermédiaires dont on peut éventuellement se passer ou à qui l'on peut préférer un autre intermédiaire par exemple. Bien souvent, au contraire, ces courtiers tentent de s'imposer comme des intermédiaires «exclusifs», que les acteurs extérieurs peuvent difficilement contourner. L'objectif de cet article est d'illustrer cet agenda de recherche établi par Bierschenk et al. (2000) à travers un cas d'étude issu d'un pays anglophone, alors que ces auteurs ont principalement appliqué leur concept au contexte de l'Afrique de l'Ouest francophone (Bierschenk et al., 2002). Nous entendons démontrer comment cette distinction heuristique entre rôle de «courtier » et rôle de gatekeeper est dans les faits incarnée par une même personne, qui en fonction des circonstances endosse l'une ou l'autre de ces postures.

\section{Zone d'étude et méthodologie}

Nous analysons ici un dispositif public particulier: le projet de réhabilitation de périmètres irrigués pour l'agriculture familiale appelé Revitalisation of Smallholder Irrigation Schemes (RESIS) et situé dans la zone du barrage Flag Boschielo de la municipalité de district de Sekhukhune en Afrique du Sud (Fig. 1). En effet, pour lutter contre la pauvreté endémique en milieu rural, notamment dans les anciens bantoustans, le gouvernement de la province du Limpopo a inauguré à la fin des années 1990 un programme public dont l'objectif était de réhabiliter les périmètres irrigués abandonnés, situés en zones de terres communales. L'instrument de politique publique principal de ce programme était un «partenariat stratégique» entre agriculteurs «intensifs » blancs et agriculteurs noirs émergents. Ce partenariat était censé prendre la forme d'une coentreprise, au sein de laquelle l'agriculteur commercial apportait son expérience, son expertise (en production, gestion, marketing) et ses capitaux, tandis que les petits agriculteurs noirs fournissaient la terre et leur allocation en eau. Cet instrument a, par la suite, été diffusé dans d'autres provinces sud-africaines.

Nous mènerons, dans cet article, une réflexion en deux temps en nous appuyant sur le matériau empirique recueilli entre 2010 et 2013 au cours d'un projet de recherche sur « la gouvernance multiniveaux dans l'accès à l'eau et au foncier pour réduire la pauvreté dans le bassin du Limpopo (Afrique du Sud, Botswana, Zimbabwe, Mozambique)». Pour le cas sud-africain, ce projet a donné lieu à une enquête de terrain auprès de groupes témoins (focus groups) organisés entre juillet et septembre 2012, puis à une vingtaine de nouveaux entretiens semi-directifs entre février et octobre 2013, principalement dans deux villages voisins de Setlaboswane 


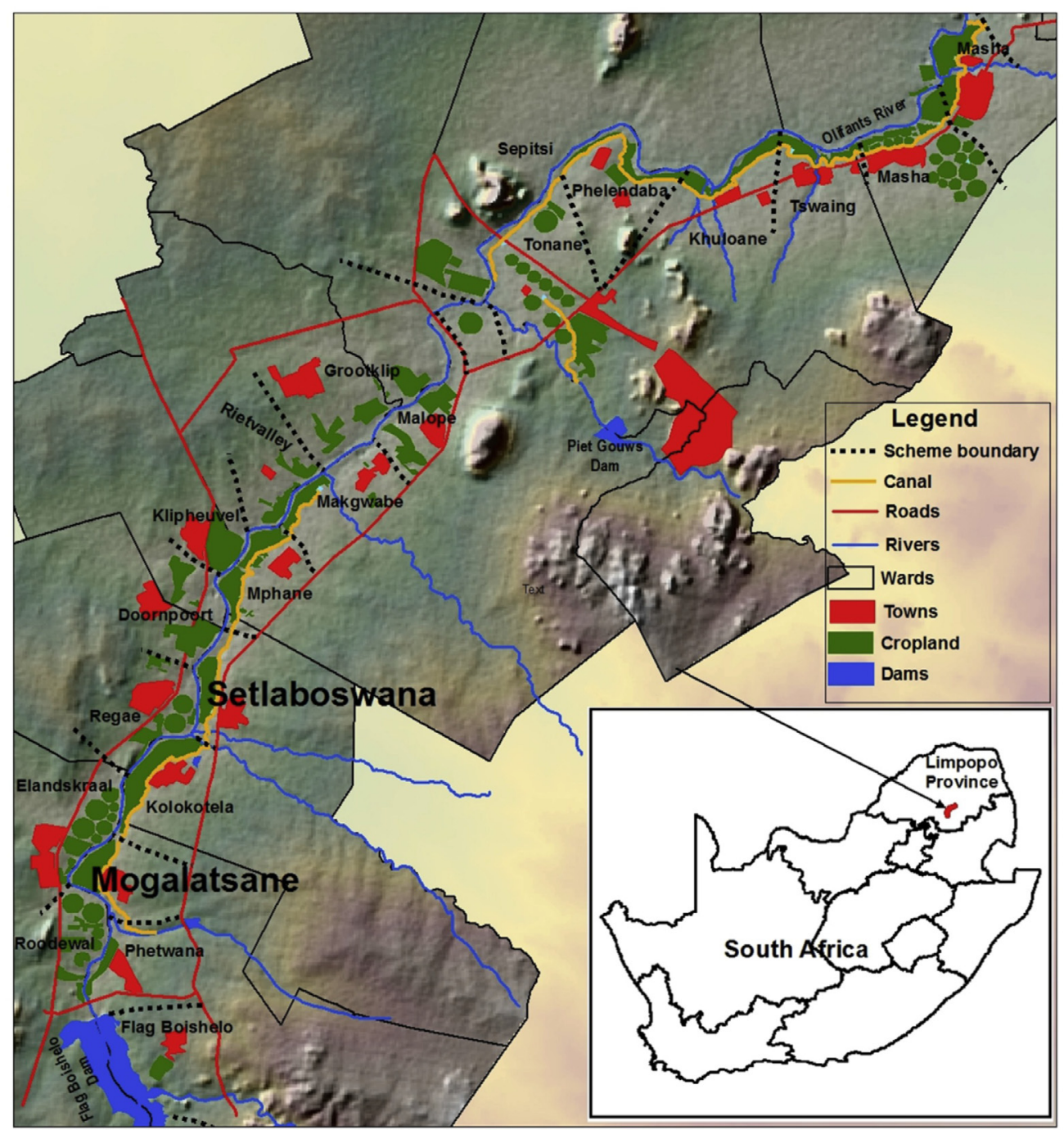

Fig. 1. Zone d'étude.

Source: Google map (Nhamo L, International Water Management Institute).

Fig. 1. Study area.

et Mogalatjane. Ces deux études de cas ont également bénéficié de résultats d'enquêtes préalables menées par un des membres de l'équipe de recherche dans les trois autres villages (Kolekotela, Phetwane et Elandskraal) situés sur le plateau du Nebo (van Koppen et al., 2015). Dans un premier temps, nous exposerons les «dommages collatéraux» de l'intervention publique, en montrant la propension de ces programmes à obtenir l'effet inverse de l'objectif recherché, soit en aggravant la situation de pauvreté soit en exacerbant les conflits locaux. Nous examinerons également leur propension à modifier les arrangements institutionnels des communautés locales. Dans un second temps, nous nous arrêterons sur l'émergence de nouveaux gardiens de l'accès aux ressources sur le terrain. Nous nous pencherons à cette occasion sur les relations qu'entretiennent figures anciennes et figures nouvelles du courtage que sont les élites traditionnelles et les comités villageois.

\section{Quand I'intervention publique alimente la pauvreté et les conflits}

Un des éléments centraux du dispositif public de réhabilitation des périmètres irrigués mis en œuvre sur le plateau du Nebo vise la promotion d'une technologie ultrasophistiquée d'irrigation par aspersion, appelée floppy sprinklers. Cette préférence des autorités publiques envers cette technologie d'irrigation spécifique a engendré un certain nombre d'effets pervers. En effet, cette technologie d'irrigation par aspersion requiert de synchroniser les activités agricoles (Setlaboswane focus group, 17 août 2012) et donc de coordonner les activités à cet effet, ce qui est perçu comme une gageure, étant donné les faibles capacités d'action collective et le niveau de conflictualité hérité des violences politiques vécues sous l'Apartheid, qui caractérisent les communautés du Sekhukhuneland (Delius, 1996). De plus, avec l'introduction 
de cette nouvelle technologie, il a nécessairement fallu procéder à un regroupement des terres en une parcelle unique. Avant cela, chaque irrigant des périmètres concernés disposait d'une parcelle très modeste, souvent moins d'un hectare. Cet arrangement concernant la fusion en une parcelle unique cause des difficultés, car il implique que l'ensemble de la communauté des irrigants - une centaine par périmètres'accorde non seulement pour coordonner les questions d'irrigation, mais aussi désormais sur le projet agricole et les cultures dans lesquelles investir. Chaque décision prise dans le cadre du projet de partenariat stratégique engage donc de fait l'ensemble des irrigants, qui devraient ainsi se réunir afin de valider chacune de ces décisions, ce qui en pratique est perçu comme irréaliste au vu du nombre d'agriculteurs à mobiliser, à intervalle régulier. De plus, le regroupement foncier dicté par la technologie d'irrigation par aspersion est vu comme irréversible; pour les chefs traditionnels, il n'est plus possible de revenir à un mode de décision individuelle dans l'administration de la terre, puisque les démarcations originelles des parcelles individuelles ont disparu (entretien, conseil exécutif traditionnel, Setlaboswane, octobre 2013). Cet état de fait modifie donc les arrangements fonciers, entraînant un basculement de l'autorité en la matière des chefs traditionnels vers les membres du conseil d'administration des comités d'irrigation, ce qui cause des tensions. En l'occurrence, cela brouille encore un peu plus les arrangements autour de la question foncière, déjà complexe à l'origine.

En effet, en Afrique du Sud, si la terre dans les zones communales appartient juridiquement à l'État, il semble qu'il faille distinguer entre règles formelles et règles informelles en la matière. Ainsi, s'il est admis que le périmètre irrigué appartient à l'État, en fait, il faut comprendre que seuls les équipements au sein de ce périmètre lui appartiennent; la terre, quant à elle, demeure la propriété de la chefferie traditionnelle. Dans les villages de Setlaboswane et Mogalatsane, il est acquis que "the chief is the only one who can allocate land for farming: in sepedi "Naga keyakgotsi" (the land belongs to the chief). [As a consequence] For all the previous farming projects that took place in the two communities, the chief's permission had to be obtained» (Bourblanc, 2013). Le personnel détaché sur site du ministère provincial de l'agriculture du Limpopo (Limpopo Department of Agriculture [LDA]) confirme cette interprétation du droit en pratique. Il souligne par exemple que lorsque des villageois sont mécontents de ne pouvoir disposer d'une parcelle de terre via l'induna du village (représentant du chef traditionnel), et qu'ils le sollicitent pour obtenir une parcelle, soit au sein du périmètre, soit en marge du périmètre le long du canal d'irrigation dont le personnel du LDA s'occupe, il se déclare invariablement dans l'incapacité d'intervenir et renvoie vers la seule autorité légitime pour attribuer un lopin de terre dans la zone, à savoir le chef traditionnel.

De plus, la question de la distribution des terres au Nebo est un sujet propice aux disputes, particulièrement à la période actuelle. Par le passé, le chef tribal avait pour habitude d'attribuer presque automatiquement un lopin de terre pour construire une habitation, ainsi qu'un lopin de terre cultivable pour chaque nouveau couple marié. Ce n'est plus le cas aujourd'hui, plus aucune terre cultivable n'étant disponible. Cette impossibilité d'allouer des terres, notamment au sein du périmètre irrigué visé par un projet de réhabilitation, nourrit des tensions en particulier générationnelles, les bénéficiaires d'un lopin de terre au sein du périmètre irrigué appartenant en général à la génération plus âgée. Dans le village voisin de Phetwane, ces personnes âgées constituent jusqu'à $83 \%$ des irrigants du périmètre (Tapela, 2008). Dans ce contexte, l'annonce du remembrement des terres au sein d'une parcelle unique dans le cadre de la réhabilitation du périmètre et de l'expérimentation d'un projet d'agriculture commerciale fut au départ accueillie favorablement par certains laissés pour compte, aux premiers rangs desquels la jeune génération du village (Mogalatjane 3rd focus group discussion, 2012; Bourblanc, 2013). Cette jeune génération constitue la moitié de la population du village, la majorité étant au chômage. Certains jeunes désœuvrés avaient donc anticipé un impact positif du développement d'un projet d'agriculture commerciale au sein du périmètre irrigué qui promettait d'employer la main-d'œuvre locale. Cette promesse ne s'est pourtant jamais matérialisée, le partenaire stratégique recrutant seulement une poignée de villageois.

Le mécontentement vis-à-vis du projet de réhabilitation ne s'arrête pas là. Des critiques se sont également élevées très tôt vis-à-vis d'un périmètre irrigué qui, pour les villageois sans terre, capterait l'attention des autorités publiques et de leurs financements, aux dépens d'autres bénéficiaires potentiels de l'aide au développement pouvant prétendre à des résultats plus probants, par exemple des porteurs de projets de jardins potagers en dehors du périmètre (backyard gardening) (Mapedza et al., 2016). Ces villageois, n'ayant pu disposer de terres au sein du périmètre, voudraient en effet continuer à pratiquer une agriculture de subsistance. Pour cela, ils ont besoin de bénéficier d'un accès à l'eau plus conséquent que celui dont ils bénéficient jusqu'à présent, à savoir un accès à l'eau potable municipale, de fait limité aux $25 \mathrm{~L}$ gratuits par jour et par personne, reconnus par la loi de réforme sur l'eau de 1998 pour couvrir les besoins humains fondamentaux. Ils réclament notamment des subventions pour pouvoir bénéficier d'un accès sécurisé - la plupart des connections étant illégales (Tapela, 2009) - et utiliser plus que les $25 \mathrm{~L}$ accordés. Le mécontentement concerne aussi certains agriculteurs qui jusque-là bénéficiaient d'un accord tacite pour cultiver un petit potager le long du canal d'irrigation et qui ont été chassés des abords du périmètre irrigué, au prétexte qu'en n'entretenant pas suffisamment leur parcelle, ils faisaient courir le risque d'un incendie qui se propagerait à l'ensemble du périmètre et détruirait le système d'irrigation ultramoderne et particulièrement onéreux (entretien avec le personnel détaché sur site chargé de la vulgarisation en matière agricole ; LDA, octobre 2013). Enfin, l'investissement dans la technologie des floppy sprinklers est source de conflits, non seulement entre titulaires d'une parcelle dans le périmètre et villageois sans terre, mais entre membres du périmètre lui-même. En effet, l'installation des floppy sprinklers a conduit à réaménager le périmètre et à exclure de fait quelques individus, dont la parcelle située aux extrémités du périmètre ne pouvait être couverte par le système fixe et aligné de l'irrigation par aspersion (entretien avec le conseil exécutif traditionnel, octobre 2013). Ainsi, il semble qu'en se focalisant uniquement sur le périmètre irrigué, l'action publique de lutte contre la pauvreté a contribué à renforcer les inégalités sur le plateau du Nebo. Par ailleurs, avec la consolidation des parcelles consécutive à la signature du partenariat stratégique, le droit 
de regard sur le foncier est désormais partagé entre État, chefferie et comité de gestion du périmètre, selon une ligne de partage des responsabilités extrêmement floue. In fine, la modification des arrangements institutionnels locaux sur le foncier apparaît comme un effet pervers de la mise en place des partenariats stratégiques.

\section{4 Élites traditionnelles et comités villageois: des courtiers rivaux}

Dans la région étudiée, le périmètre irrigué est désormais administré par une structure coopérative (en l'occurrence l'Irrigation Farmers Cooperative Ltd.). La création d'une structure unique de gestion était, en effet, une condition imposée par le ministère provincial de l'agriculture du Limpopo au moment de la mise en place des accords de partenariat stratégique. L'existence d'un tel comité était censée faciliter la mobilisation des irrigants du périmètre et donc la mise en œuvre de ces accords. Depuis les années 1980 et 1990 , de telles structures associatives sont une préconisation standard diffusée au niveau international par les politiques d'inspiration néolibérale prescrites par la Banque mondiale, visant le retrait de l'État des structures de gestion des périmètres irrigués et leur transfert aux communautés locales. Dans l'Afrique du Sud des premières années postApartheid, la promotion de telles coopératives ou comités d'irrigation a surtout été l'occasion pour le nouveau régime démocratique en place d'affaiblir le pouvoir des élites traditionnelles, accusées de collaboration avec le régime d'Apartheid. La mise en place d'un tel comité viendrait ainsi en quelque sorte concurrencer les structures de décision établies par la hiérarchie tribale.

Pour autant, bien que créés à l'initiative des nouvelles élites démocratiques sud-africaines, ces comités sont loin d'être des intermédiaires passifs (Gluckman et al., 1949) ou des relais dociles des autorités publiques. En premier lieu, il n'est pas certain que ces nouvelles structures soient parvenues à se substituer aux figures historiques du courtage que sont les chefs tribaux. On constate en effet, dans la pratique, des identités largement entremêlées entre ces deux groupes. À Setlaboswane, on relève par exemple que certains membres de la direction $\mathrm{du}$ nouveau comité en exercice (Irrigation Committee's Governing Board) ont des liens de parenté avec les conseillers des chefs traditionnels ou que d'anciens membres de la direction deviennent des membres de plein droit du conseil exécutif traditionnel une fois leur mandat terminé (enquête de terrain, octobre 2013). Il n'y a donc pas de séparation stricte entre les deux structures organisationnelles, ce qui semble démontrer la capacité de la chefferie traditionnelle à phagocyter les structures de représentation concurrentes. En ce sens, nous aurions tort d'opposer au sein du village les deux formes d'organisation: celle, héritée, centrée sur la chefferie traditionnelle et celle, élective, centrée autour du comité d'irrigation, leur relation ne se résumant pas à cet antagonisme des origines. Cela confirme, comme le montrent les développements les plus récents de la littérature sur les courtiers, les marges de manœuvre dont disposent ces acteurs du courtage sur le terrain.

Cette filiation tribale n'est cependant pas l'attribut principal mis en avant par les villageois pour sélectionner les membres de la direction des comités d'irrigation. En premier lieu, ces représentants sont censés être élus parmi l'assemblée des agriculteurs du périmètre irrigué. En pratique, cette condition n'est pas toujours respectée. Ce qui est mis en avant, ce sont en fait des qualités telles que la maîtrise de l'anglais, les capacités communicationnelles et rédactionnelles du représentant, la facilité avec laquelle il est capable d'interagir avec l'extérieur du village, bien plus que le fait de posséder ou non des terres dans le périmètre irrigué. En règle générale, les membres de ce comité sont donc plutôt jeunes et disposent d'une expérience en dehors du village, qu'ils ont quitté par le passé pour chercher du travail en milieu urbain. Le rôle d'intermédiaire, en particulier d'interface et de relais vers l'extérieur, des membres de la direction du comité est donc particulièrement valorisé et sollicité. Pour se faire élire, la stratégie de ces aspirants courtiers est de se présenter comme tel auprès des villageois, comme traducteurs de leurs demandes et aspirations.

Pourtant, il semble qu'une fois en poste, ce soit surtout le rôle de nouveau gatekeeper que les membres de la direction de ces comités d'irrigation privilégient, et cela invariablement, au gré des comités successifs. En fonction des contextes, ce rôle est endossé soit en concurrence directe des autorités traditionnelles, soit envisagé comme un checkpoint supplémentaire au sein d'un système de contrôle de l'accès aux ressources organisé au profit des élites tribales. À Mogalatjane, un nouveau comité s'est ainsi autoproclamé, en dénonçant la collusion entre l'ancien comité et les élites traditionnelles soupçonnés de malversation dans le cadre de la mise en œuvre du partenariat stratégique, ce qui a débouché sur une crise politique au sein du village. Même dans le cas de Setlaboswane, où le comité est réputé être en bons termes avec les élites traditionnelles, deux circuits de décision semblent désormais coexister, si l'on en juge par la divergence d'interprétation quant aux obligations et responsabilités des uns et des autres. Ainsi, la dizaine de personnes qui composent la direction du comité d'irrigation estiment, hors la présence du conseil exécutif traditionnel, qu'elles sont la seule structure légitime officiant en tant qu'intermédiaire entre villageois et intervenants extérieurs au village, pour toutes les questions relatives au périmètre irrigué et au projet de partenariat stratégique (entretien avec le Président du conseil d'administration du comité de gestion du périmètre irrigué, Setlaboswane, octobre 2013). Cette direction se positionne ainsi comme l'unique point d'entrée pour toute demande extérieure. À ce titre, elle récuse toute tentative de contourner le comité afin de traiter directement avec le chef traditionnel, comme cela fut parfois le cas avec le personnel sur site du ministère provincial de l'agriculture à Mogalatjane. De son côté, au contraire, le conseil traditionnel reproche à la direction du comité de ne pas toujours le tenir informé de toutes les affaires concernant le périmètre irrigué et de leurs développements (enquête de terrain, octobre 2013). Si la chefferie traditionnelle est prête à admettre que le leadership sur toutes les questions relatives au périmètre irrigué se partage entre élites traditionnelles et membres du conseil de direction du comité d'irrigation, elle conçoit malgré tout ce partage selon une ligne de commande verticale. Autrement dit, selon l'interprétation des élites traditionnelles, le comité d'irrigation peut être l'interlocuteur en première ligne sur toutes les questions de gestion 
quotidienne du périmètre et les aspects techniques du projet, mais il doit nécessairement en référer à la chefferie traditionnelle dès lors qu'une décision doit être prise, décision devant s'effectuer sous le contrôle strict de la hiérarchie traditionnelle. Ainsi, en fonction des contextes, comité d'irrigation et chefferie traditionnelle oscillent entre des stratégies de courtage hégémonique ou de courtage exclusif.

Une constante s'observe également, relative aux allégations de corruption. On savait ces pratiques de corruption très répandues parmi les élites tribales depuis la destitution du chef Sekhukhune et son remplacement par des chefs à la solde du régime d'Apartheid (van Koppen et al., 2015). Elles sont plus surprenantes s'agissant des comités d'irrigation, une innovation qui se voulait au départ démocratique, promouvant une structure élue par les pairs, face à la charge héritée des chefs traditionnels. D'une manière générale, les villageois reprochent aux membres de la direction de ces comités de décider pour l'ensemble des irrigants sans nécessairement réunir à chaque fois l'ensemble des irrigants du périmètre ni les tenir informés des décisions cruciales prises. Cela a pour effet d'alimenter la défiance envers ces directions dont les villageois critiquent immanquablement le manque de transparence et le peu d'empressement à rendre des comptes. Favorisées par la complexité de l'accord financier et le caractère relativement abscons du projet de partenariat stratégique, les tentations d'un comportement opportuniste sont permanentes et liées aux gains matériels personnels qui peuvent être retirés de ces contrats. Du fait de ces critiques récurrentes et malgré les renversements successifs de comité, il apparaît donc que ces travers constituent une dimension structurelle attachée à la position de courtier/gatekeeper.

\section{Conclusion}

En analysant la mise en cuvre au niveau local des programmes publics de lutte contre la pauvreté rurale en Afrique du Sud, nous avons souligné un certain nombre de dysfonctionnements de ces programmes. À tel point que les périmètres irrigués se retrouvent aujourd'hui à l'abandon, en dépit des nombreux investissements dont ils ont fait l'objet depuis la mise en œuvre du programme public RESIS. On peut même dire que ces programmes publics, en altérant les arrangements institutionnels dans les lieux où ils interviennent, provoquent dans bien des cas de nouveaux conflits. En outre, les décideurs publics se montrent aveugles au rôle joué par des acteurs-clés de l'application de ces programmes que sont les courtiers en développement. Nous avons souhaité illustrer au travers de cet article le double rôle de médiateur et de gardien de l'accès aux ressources qu'endossent les acteurs de ce jeu de courtage. Par ailleurs, dans la mesure où il existait déjà une offre de courtage présente sur le terrain en la figure de la chefferie traditionnelle, nous nous sommes attachés à comprendre les relations que ces comités villageois pouvaient entretenir avec celle-ci, ce que peu de travaux ont cherché à faire. In fine, en combinant le concept de broker issu de l'anthropologie politique anglo-saxonne, celui de gatekeeper issu des travaux en science politique et celui de courtier issu de l'anthropologie du développement francophone, on voit apparaître un acteur censé être le représentant des villageois et le traducteur de demandes provenant à la fois de l'intérieur et de l'extérieur du village, mais qui, dans sa relation aux élites tribales notamment - nonobstant les liens de parenté existant parfois - présente un mélange hybride de collaboration rivale et de tentative de contrôle hégémonique, voire exclusif, de l'accès aux ressources du village.

\section{Références}

Bailey. 1960. Tribe, caste, and nation: a study of political activity and political change in highland Orissa. Manchester: Manchester University Press.

Bayart J-F, Mbembe A, Toulabor C. 1992. Le politique par le bas en Afrique noire. Paris: Karthala, 228 p.

Beresford. 2015. Power, patronage, and gatekeeper politics in South Africa. African Affairs 114(455): 226-248.

Bierschenk T, Chauveau J-P, Olivier de Sardan P, eds. 2000. Courtiers en développement : les villages africains en quête de projets. Paris: Karthala, $328 \mathrm{p}$.

Bierschenk T, Chauveau J-P, Olivier de Sardan J-P. 2002. Local development broker in Africa. The rise of a new social category. Institut für Ethnologie und Afrikastudien, Johannes GutenbergUniversität, working paper No. 13, $46 \mathrm{p}$.

Boissevain J. 1969. Patrons as Brokers. Sociologische Gids 16: 379 386.

Boissevain J. 1974. Friends of Friends. Networks, Manipulators and Coalitions. Oxford: Basil Blackwell.

Bourblanc M. 2013. Pro-poor policies for water and land access in Flag Boshielo Irrigation Scheme: consolidated South African report. Cirad-University of Pretoria, Report for Challenge Program Water and Food (CPWF)-Limpopo Basin Development Challenge (LBDC), CGIAR Consortium of International Agricultural Research Centres, $54 \mathrm{p}$.

Cooper F. 2002. Africa since 1940: the past of the present. Cambridge: Cambridge University Press, $216 \mathrm{p}$.

Darbon D. 2004. Pour une socioanthropologie de l'administration en Afrique II. Retour méthodologique à propos d'un article de JeanPierre Olivier de Sardan. Politique Africaine 96: 163-173.

Delius P. 1996. A lion amongst the cattle: reconstruction and resistance in the Northern Transvaal. Johannesburg: Ravan Press.

Evans L. 2012. South Africa's Bantustans and the dynamics of 'decolonisation': reflections on writing histories of the homelands. South African Historical Journal 64(1): 117-137.

Ferguson J. 1990. The anti-politics machine: 'development', depoliticization, and bureaucratic power in Lesotho. Cambridge: Cambridge University Press, 336 p.

Gluckman M, Mitchell C, Barnes JA. 1949. The village headman in British Central Africa. Africa 19: 89-106.

Mair L. 1968. Anthropology and social change. London, Athlone: London School of Economics Monographs on Social Anthropology, No. 38 .

Mamdani M. 1996. Citizen and subject. Contemporary Africa and the legacy of late colonialism. Kampala/London: James Currey, 368 p.

Mapedza E, Van Koppen B, Sithole P, Bourblanc M. 2016. Joint Venture Schemes in Limpopo Province and their outcomes on smallholder farmers Livelihoods. Physics and Chemistry of the Earth 92: 92-98.

Mosse D, Lewis D, eds. 2006. Development brokers and translators. Bloomfield: Kumarian, $251 \mathrm{p}$.

Olivier de Sardan J-P. 2007. De la nouvelle anthropologie du développement à la socio-anthropologie des espaces publics africains. Revue Tiers Monde 191(3): 543-552. 
Smith A. 2013. L'analyse des politiques publiques. In: Balzacq T, Ramel F, eds. Traité des relations internationales. Paris : Presses de Sciences Po, pp. 439-466.

Tapela B. 2009. Assessment of formal and informal hydraulic property rights creation at local level. Case study of Phetwane and selected Arabie/Olifants communities in Limpopo province, South Africa. Plaas, University of Western Cape, Report for CGIAR Challenge programme 66, Part 2 of the consolidated report, 81Plaasp.
Tapela BN. 2008. Livelihoods in the wake of agricultural commercialisation in South Africa's poverty nodes: insights from small-scale irrigation schemes in Limpopo Province. Development Southern Africa 25(2): 181-198.

van Koppen B, Tapela B, Mapedza E. 2015. Gender, rights and the politics of productivity: the case of the Flag Boshielo Irrigation Scheme, South Africa. In: Hellum B, Kameri-Mbote P, van Koppen B, eds. Water is life: Women's Human Rights in National and Local Water Governance in Southern and Eastern Africa. Harare: Weaver Press, pp. 535-574.

Citation de l'article : Bourblanc M, Ducrot R. 2018. Courtiers en développement et contrôleurs d'accès : deux figures incontournables des politiques de lutte contre la pauvreté rurale en Afrique du Sud. Cah. Agric. 27: 55006. 